\title{
Mixed Mode Partitioning of Beam-Like Geometries: A Damage Dependent Solution
}

\author{
M. Conroy ${ }^{\mathrm{a}}$, A.J. Kinloch ${ }^{\mathrm{b}}$, J.G. Williams $^{\mathrm{b}}$, A Ivankovic ${ }^{\mathrm{a}, *}$ \\ ${ }^{a}$ School of Mechanical and Materials Engineering, University College Dublin, Belfield, Dublin 4, Ireland \\ ${ }^{b}$ Imperial College London, Mechanical Engineering Department, Exhibition Road, London SW7 2AZ, UK
}

\begin{abstract}
The current work studies the fracture mode partition in beam-like geometries as a function of cohesive properties. It is observed that the mode mixity exhibits a unique dependence on the cohesive zone length scale, where the lower and upper bounds are given by the local and global partitioning. Based on this observed unique dependency, a new semi-analytical cohesive analysis (SACA) is proposed for partitioning. This partitioning method is applied to previously conflicting experimental data in the literature, and physically consistent results are obtained in each case, suggesting that this novel SACA method can be used to obtain accurate mixed mode partitioning now always.
\end{abstract}

Keywords: Cohesive Zone Model, Mixed Mode Fracture, Partitioning, Fracture Test Methods

\section{Introduction}

It has been widely documented that the toughness of adhesive joints and composite laminates can vary considerably depending on the mode of loading [1, 2, 3, 4. In many of these cases, the mode I energy release rate (ERR), $G_{I C}$, is shown to be the most critical, and hence is a conservative value when used in design. However, Dillard et al. 3] highlighted a number of cases where the lowest measured ERR, $G_{C}$, occurs at a certain mixed mode I/II loading. To eliminate the risk of non-conservative design, and also avoid over design, it is important to characterise joint toughness over a full range of mixed mode loadings ranging from pure mode I (tensile) to pure mode II (in-plane shear). Test methods for fracture characterisation of the pure modes I and II have been well established [5, 6. However, while a number of options exist for mixed mode I/II testing [7, 8, 9, it is an area of considerable contentiousness and uncertainty, in particular regarding the mode decomposition of asymmetric specimens.

Beam-like geometries as shown in fig. 1 have been the main basis for the development of analytical mixed mode partitioning theories, and will be the focus of the current study. The mode mixity $\left(G_{I I} / G\right)$ is defined here as the ratio of the energy release rate in mode II $\left(G_{I I}\right)$ to the total energy release rate $(G)$, where $G=G_{I}+G_{I I}$. Williams [10] proposed a fully analytical approach for decomposing ERRs into mode I and mode II components based on classical beam theory, without considering the details of local stress and strain distributions at the crack tip. This approach is often referred to as the global approach. Yin and Wang [11] and Suo and Hutchinson [12] proposed a solution based on the decomposition of stress intensity factors (SIFs) by considering local conditions at the crack tip. Thus, in contrast to the Williams 10 global partitioning approach, this approach assumes the presence of a $K$ dominant region and is commonly referred to as the local partitioning approach. The predicted mode partition from both the local and global approaches are independent of material properties and depend only on $\gamma$ and $k$, where $\gamma$ is the ratio of beam heights and $k$ is the ratio of applied moments as defined in fig 1 . The predicted partitions from both methods agree

\footnotetext{
* Corresponding author

Email address: alojz.ivankovic@ucd.ie (A Ivankovic)
} 
only when the crack is located centrally $\left(h_{1}=h_{2}\right)$, as shown in fig. 2(a) where the predicted mixed mode partitions $\left(G_{I I} / G\right)$ from the local and global approaches are plotted as a function of the applied moment ratio $k$ for symmetric specimens. In cases where $h_{1} \neq h_{2}$, the differences between the local and global approaches are significant, as is evident in fig. $2(\mathrm{~b})$ where the predicted mixed mode partition $\left(G_{I I} / G\right)$ from both approaches are plotted as a function of the beam heigh ratio $\gamma$ for $k=0$.

Numerous experimental studies have investigated the accuracy of both the local and global approaches. Hashemi et al. 11 carried asymmetric tests on carbon epoxy composite and applied both local and global partitioning. It was found that the global approach produced a more physical failure locus and it was proposed that this was likely due the fracture process zone being larger than the relatively small $K$ dominant region (often $\leq 1 \%$ of smallest beam thickness [13]). Davidson et al. [14] also carried out asymmetric tests on carbon epoxy composite and compared the resulting failure loci, obtained via local and global partitioning, to the already measured true failure locus obtained using symmetric specimens. It was found that neither the local nor global approach predicted the mode partition adequately. Ducept et al. [15] undertook a similar study on glass reinforced epoxy composite and found that the local partitioning accurately predicted the symmetrically measured failure locus.

It is evident that considerable uncertainty still surrounds the area of mixed mode partitioning. The goal of the current work is to numerically simulate fracture in common asymmetric fracture test geometries with the use of a cohesive zone model and then carry out a parametric study on the effect of cohesive and substrate properties on the mode mixity. As all of the substrate and cohesive properties are linked to the numerical cohesive zone length, $l_{c z}$, the effect of the size of the developed cohesive zone length on the mode mixity $\left(G_{I I} / G\right)$ is of particular interest and is studied in detail. Based upon the numerical findings from this study, a new semi-analytical cohesive analysis (SACA) scheme is proposed for partitioning mixed mode fractures. The novelty of this SACA scheme is that it accounts for damage state at the crack tip by incorporating a cohesive length scale parameter, $l_{n d}$. This damage dependent partitioning scheme is then applied to previously conflicting experimental data [1, 15] and the success of the new partitioning scheme is critically assessed.

\section{Analytical Partitioning}

\subsection{Global Partitioning}

Using beam theory, the total energy release rate for an increment of crack growth in fig. 1 can be shown to be:

$$
G=\frac{6}{B^{2} E}\left(\frac{M_{1}^{2}}{h_{1}^{3}}+\frac{\left(k M_{1}\right)^{2}}{h_{2}^{3}}-\frac{M_{1}^{2}(1+k)^{2}}{\left(h_{1}+h_{2}\right)^{3}}\right)
$$

where $E$ is the beam modulus in the bending direction and $B$ is the out of plane width. Williams $[10$ proposed that the applied moments could be decomposed into components which caused pure mode I $\left(M_{I}\right)$ and pure mode II $\left(M_{I I}\right)$. By proposing that pure mode I occurs when moments are equal and opposite $\left(-M_{1}=k M_{1}\right)$, and that pure mode II occurs when the beam curvatures are equal $\left(M_{1} / E I_{1}=k M_{1} / E I_{2}\right)$, it is possible to represent the applied moments as a combination of the decomposed moments as:

$$
\begin{aligned}
& M_{1}=M_{I I}-M_{I} \\
& k M_{1}=\psi M_{I I}+M_{I}
\end{aligned}
$$

where $\psi=\left(h_{2} / h_{1}\right)^{3}$. The resultant mode I and mode II energy release rates can be found directly by substituting eqs. 2 into eq. 1 as no cross product term containing $M_{I} M_{I I}$ is produced. The resulting decomposed energy release rates are given by:

$$
\begin{gathered}
G_{I}=\frac{6 M_{I}^{2}(1+\psi)}{B^{2} E h_{2}^{3}} \\
G_{I I}=\frac{18 M_{I I}^{2} h_{2}(1+\psi)}{B^{2} E h_{1}^{2}}
\end{gathered}
$$




\subsection{Local Partitioning}

In the local approach [12, the partitioning of eq. 1 is carried out by obtaining the singular field stress distributions at the crack tip and solving for the decomposed stress stress intensity factors $K_{I}, K_{I I}$. Using the relation between energy release rate and stress intensity factors for homogeneous isotropic materials i.e.

$$
G=\frac{\left(K_{I}^{2}+K_{I I}^{2}\right)}{E}
$$

the decomposed energy release rates are expressed as:

$$
\begin{aligned}
G_{I} & =\frac{1}{E}\left(\frac{P}{\sqrt{2 h_{1} U}} \cos (\omega)+\frac{M}{\sqrt{2 h_{1}^{3} V}} \sin (\omega+\phi)\right)^{2} \\
G_{I I} & =\frac{1}{E}\left(\frac{P}{\sqrt{2 h_{1} U}} \sin (\omega)-\frac{M}{\sqrt{2 h_{1}^{3} V}} \cos (\omega+\phi)\right)^{2}
\end{aligned}
$$

where $M$ and $P$ are linear combinations of the applied loads. In the particular case of the loading shown in fig. 1, these are defined as:

$$
\begin{gathered}
P=-C_{2} \frac{(1+k) M_{1}}{h_{1}}, \quad M=M_{1}-C_{3}(1+k) M_{1} \\
C_{2}=\frac{6}{(1 / \gamma+1)^{3}}, \quad C_{3}=\frac{1}{(1 / \gamma+1)^{3}}
\end{gathered}
$$

and $U, V$ and $\phi$ are geometric factors defined by:

$$
\begin{aligned}
& U=\frac{1}{1+4 \gamma+6 \gamma^{2}+3 \gamma^{3}}, \quad V=\frac{1}{12\left(1+\gamma^{3}\right)} \\
& \phi=\sin ^{-1}\left(\frac{6 \gamma^{2}(1+\gamma)}{\sqrt{U V}}\right)
\end{aligned}
$$

$\omega$, which is a function of $\gamma$, can not be obtained analytically and is extracted from a rigorous numerical solution of the case in which $\gamma \rightarrow 0$, and then linearly approximated over the range $0 \leq \gamma \leq 1$. The value of $\omega$ at $\gamma=1$ is known because the mode partition is exact in this case. The proposed linear approximation is given by:

$$
\omega=52.1-3 \gamma
$$

\section{Numerical Partitioning of Cohesive Zones}

Cohesive zone models have been used extensively to simulate fracture process zones since the concept was first introduced by Dugdale [16] and Barenblatt [17. A typical fracture process zone is shown in fig. 3.

The fracture process zone consists of a crack tip located at $a$, where cohesive stresses drop to zero, and a damage zone tip located at $a^{*}$, which occurs when the cohesive strength, $t_{C}$, is reached. The distance between $a$ and $a^{*}$ is the cohesive zone length $\left(l_{c z}\right)$. When the damage region is propagating in a self similar manner under monotonic loading between linear elastic beams, the concept of energy release rate $G$ can be related to the path independent $J$ integral proposed by Rice [18, which can be expressed as:

$$
G=J_{\Gamma_{e x t}}=J_{\Gamma_{c o h}}+J_{\Gamma_{t i p}}
$$

for the fracture process zone shown in figure $3 . \Gamma_{e x t}$ is a path going continuously from a point on the lower fully cracked face to the upper fully cracked face around the crack tip and fully encapsulating the cohesive region, $\Gamma_{c o h}$ corresponds to a path along the upper and lower surfaces of the active cohesive zone and $\Gamma_{t i p}$ corresponds to an infinitely small path surrounding the crack tip. The $J$ integral is computed from:

$$
J=\int_{\Gamma}\left(\int_{0}^{\epsilon} \sigma_{i j} d \epsilon_{i j} d y-\sigma_{i j} n_{j} \frac{\partial u_{i}}{\partial x} d s\right)
$$


where $\sigma_{i j}, \epsilon_{i j}$ are the components of the stress and strain tensor respectively, $u_{i}$ the displacement vector, $n_{j}$ the outwards normal to the path, and $s$ is the distance along the path. By calculating the $J$ integral externally along $\Gamma_{e x t}$, it is possible to account for energy contributions from the cohesive surfaces $\left(J_{\Gamma_{\text {coh }}}\right)$ and crack tip singularity $\left(J_{\Gamma_{t i p}}\right)$, and this can be applied regardless of whether there is a cohesive region present or not. Ishikawa et al. [19. proposed a method for decomposing the $J$ integral into mode I and mode II components. This is achieved by considering symmetric integration paths about the crack plane. e.g. paths containing $P$ and $P^{\prime}$ in fig. 3, and decomposing the stress, strain and displacement fields into symmetric (causing mode I) and antisymmetric (causing mode II) components as:

$$
\begin{aligned}
\sigma_{i j} & =\sigma_{i j}^{I}+\sigma_{i j}^{I I}=\frac{1}{2}\left[\begin{array}{cc}
\sigma_{11}+\sigma_{11}^{\prime} & \sigma_{12}-\sigma_{12}^{\prime} \\
\sigma_{12}-\sigma_{12}^{\prime} & \sigma_{22}+\sigma_{22}^{\prime}
\end{array}\right]+\frac{1}{2}\left[\begin{array}{cc}
\sigma_{11}-\sigma_{11}^{\prime} & \sigma_{12}+\sigma_{12}^{\prime} \\
\sigma_{12}+\sigma_{12}^{\prime} & \sigma_{22}-\sigma_{22}^{\prime}
\end{array}\right] \\
\epsilon_{i j}=\epsilon_{i j}^{I}+\epsilon_{i j}^{I I} & =\frac{1}{2}\left[\begin{array}{ll}
\epsilon_{11}+\epsilon_{11}^{\prime} & \epsilon_{12}-\epsilon_{12}^{\prime} \\
\epsilon_{12}-\epsilon_{12}^{\prime} & \epsilon_{22}+\epsilon_{22}^{\prime}
\end{array}\right]+\frac{1}{2}\left[\begin{array}{cc}
\epsilon_{11}-\epsilon_{11}^{\prime} & \epsilon_{12}+\epsilon_{12}^{\prime} \\
\epsilon_{12}+\epsilon_{12}^{\prime} & \epsilon_{22}-\epsilon_{22}^{\prime}
\end{array}\right] \\
u_{i}=u_{i}^{I}+u_{i}^{I I} & =\frac{1}{2}\left[\begin{array}{l}
u_{1}+u_{1}^{\prime} \\
u_{2}-u_{2}^{\prime}
\end{array}\right]+\frac{1}{2}\left[\begin{array}{c}
u_{1}-u_{1}^{\prime} \\
u_{2}+u_{2}^{\prime}
\end{array}\right]
\end{aligned}
$$

These decomposed stresses, strains and displacements are substituted directly into eq. 10 to obtain the mode decomposed $J$ integral components $\left(J_{I}, J_{I I}\right)$, which equate to the mode I and mode II ERR components $\left(G_{I}, G_{I I}\right)$.

$$
\begin{aligned}
J_{I} & =G_{I}=\int_{\Gamma_{e x t}}\left(\int_{0}^{\epsilon} \sigma_{i j}^{(I)} d \epsilon_{i j}^{(I)} d y-\sigma_{i j}^{(I)} n_{j} \frac{\partial u_{i}^{(I)}}{\partial x} d s\right) \\
J_{I I} & =G_{I I}=\int_{\Gamma_{e x t}}\left(\int_{0}^{\epsilon} \sigma_{i j}^{(I I)} d \epsilon_{i j}^{(I I)} d y-\sigma_{i j}^{(I I)} n_{j} \frac{\partial u_{i}^{(I I)}}{\partial x} d s\right)
\end{aligned}
$$

In cases where the fracture process zone is fully developed and the singularity is fully suppressed i.e. $J_{\Gamma_{t i p}}=$ 0, the mode decomposed ERR components can be found directly by integrating over the cohesive surfaces as $J_{\Gamma_{e x t}}=J_{\Gamma_{c o h}}$. The resulting expression can be expressed in terms of known cohesive zone quantities as:

$$
\begin{aligned}
& G_{I}=\int_{\Gamma_{c o h}^{+}} t_{N} \frac{\partial \delta_{N}}{\partial x} d s \\
& G_{I I}=\int_{\Gamma_{c o h}^{+}} t_{S} \frac{\partial \delta_{S}}{\partial x} d s
\end{aligned}
$$

where $t_{N}, t_{S}, \delta_{N}$ and $\delta_{S}$ are the normal and shear cohesive tractions and opening displacements respectively. $\Gamma_{c o h}^{+}$indicates that the integration only needs to be performed over the top surface, which arises due to the symmetry of stresses on the upper and lower cohesive surfaces.

\section{Numerical Solutions}

\subsection{Test Case Setup}

The mode mixity in the asymmetric fixed ratio mixed mode (AFRMM) (fig. 4(a) and the asymmetric double cantilever (ADCB) (fig. 4(b) test geometries are studied as a function of cohesive properties in this work. In each test case, the beam geometry is meshed with a regular grid of $2 \mathrm{D}$ plane strain, 8 node quadratic elements in Abaqus v 6.11. The beam material is assumed linear elastic and isotropic, with a Young's modulus of $50 \mathrm{GPa}$ and a Poisson's ratio of 0.38. Loading is achieved through a fixed rotation, which is applied to a rigid surface that is tied to the end of the loaded beam. A rotation boundary condition is preferred as it induces a pure bending moment and also allows the crack to propagate in a numerically stable manner when the damage region is fully developed. This boundary condition also mimics the pure moment loading that is assumed in the local and global analytical analyses. Cohesive elements are inserted 
along the crack plane in the undamaged region as indicated in fig 4 Mesh density is chosen to ensure at least 10 cohesive elements are present in the fully developed damage region, as recommended by Turon et al. [20]. Two cohesive zone formulations are used to model the AFRMM test case for comparative purposes. The first cohesive zone, which is inbuilt into Abaqus, is a coupled linear-softening formulation proposed by Camanho et al. 21. The second cohesive zone model is an uncoupled Dugdale formulation developed by Yang et al. [22, and is implemented as a user element (UEL) subroutine in Abaqus. The cohesive zone formulations are represented graphically in fig. 5 and the details, along with the explanations of fig. 5, are given in $\mathrm{A}$. The particular test case properties for both the AFRMM and ADCB test cases are as follows:

\subsubsection{AFRMM}

The geometry, loading and boundary conditions used in the AFRMM test case can be seen in fig. 4(a). Three different $\gamma\left(\gamma=h_{1} / h_{2}\right)$ ratios are simulated corresponding to $0.1,1$, and 10 . In each of the test cases, unless otherwise stated, the mode I and mode II cohesive toughnesses $\left(G_{I C}, G_{I I C}\right)$ are set equal (i.e. $G_{C}=G_{I C}=G_{I I C}$ ), as are the normal and shear cohesive strengths (i.e. $t_{C}=t_{N C}=t_{S C}$ ). Therefore, along with the beam height ratio, the only properties which are varied in this study are the cohesive strength $t_{C}$ and cohesive toughness $G_{C}$. For each $\gamma$ ratio, the cohesive toughness is set to values of 200 and $3000 \mathrm{~J} / \mathrm{m}^{2}$, and for each of these toughness values, three different cohesive strengths are simulated, corresponding to $20 \mathrm{MPa}, 45 \mathrm{MPa}$ and $95 \mathrm{MPa}$. For each test geometry, an initial test case is carried out where the cohesive region is replaced by a perfect bond between the substrates. This allows a $K$ dominant region to develop at the crack tip and gives a bounding solution corresponding to zero damage development; this solution is referred to as the elastic solution. Two additional cases are also carried out to investigate the effect on the mode partition when $G_{I C} \neq G_{I I C}$. This was only possible with the uncoupled Dugdale cohesive zone model as the coupled linear-softening cohesive zone suffers from thermodynamic inconsistencies under variable mixed mode loading when pure mode toughnesses are unequal [23, 24. The additional cases correspond to $\left(\gamma=0.1, G_{I C}=500 \mathrm{~J} / \mathrm{m}^{2}, G_{I I C}=3000 \mathrm{~J} / \mathrm{m}^{2}, t_{N C}=t_{S C}=45 \mathrm{MPa}\right)$ and $\left(\gamma=10, G_{I C}=200 \mathrm{~J} / \mathrm{m}^{2}, G_{I I C}=800 \mathrm{~J} / \mathrm{m}^{2}, t_{N C}=t_{S C}=95 \mathrm{MPa}\right)$.

\subsection{2. $A D C B$}

The geometry, loading and boundary conditions used in the ADCB test case can be seen in fig. 4(b). Two different $\gamma$ ratios are simulated, corresponding to 0.2 and 5. In this test case, only the linear-softening cohesive zone is used. As before, two toughness values of 200 and $3000 \mathrm{~J} / \mathrm{m}^{2}$ are simulated at each $\gamma$ ratio, and for each of these toughness values, three different cohesive strengths are simulated, corresponding to 20 $M P a, 45 M P a$ and $95 M P a$.

\subsection{Post Processing}

For each case, with the exception of the elastic runs, the rotational loading is increased until steady state fracture occurs. At this point, the mode I and mode II ERR components are obtained by integrating over the cohesive surfaces using eq. 13. The cohesive zone length, $l_{c z}$, as defined in fig. 3 and section 3 . is also recorded in each case. For the elastic cases where the cohesive zone is omitted, a fixed rotation is increased until a moment of $50 \mathrm{~N} / \mathrm{m}$ develops in the loaded arm. At this point the mode I and mode II ERR components are calculated using the remote decomposed integrals given by eq. 12 .

\subsection{Results}

\subsubsection{AFRMM}

The numerical partitioning of the AFRMM test case, modelled with the coupled linear-softening CZM, is presented in fig. 6(a), and compared to local and global partitioning theories. For all height ratios, the elastic partitioning solution is in close agreement with the local partitioning. This is expected given the singular nature of the crack tip when no cohesive zone is present. When the cohesive zone is present, the partition of energies does not in general agree with either the local or the global solution. For $\gamma=1$, both the local and the global partitioning theories agree and the cohesive zone solutions accurately predict this partition. When $\gamma \neq 1$, the cohesive zone solutions predict that the partition lies in between the local 
and the global partitioning. For high strengths and low toughnesses (i.e. small cohesive zone length), the predicted mode partition lies closer to the local partitioning solution and for low strengths and high toughnesses (large cohesive zone length), the predicted mode partition lies closer to the global partitioning solution. Considering the role of $\gamma$ in all cases, the solutions lie much closer to the global solution for $\gamma=10$, compared to $\gamma=0.1$. This shift towards the global solution for $\gamma=10$ may be attributed to two main factors. Firstly, for $\gamma=10$, the loading is mode II dominant, which results in higher cohesive zone lengths compared to the mode I dominated loading for equivalent cohesive properties [25. Secondly, the smallest characteristic dimension, which is $0.3 \mathrm{~mm}$ in the $\gamma=10$ case compared to $3 \mathrm{~mm}$ in the $\gamma=0.1$ case, also plays an important role as the range of the $K$ dominant field scales with the smallest characteristic dimension. This means that the range of the $K$ dominant field is smaller in the $\gamma=10$ case and therefore the mode mixity will deviate further away from the local solution in the $\gamma=10$ case for similar cohesive zone lengths.

The corresponding results from the uncoupled Dugdale CZM are presented in fig. 6(b)] The same combinations of cohesive properties are used in this test case for $\gamma=1,10$, with the exception of three cases where convergence was not obtained. These cases occurred at each cohesive strength when $G_{C}=3000 \mathrm{~J} / \mathrm{m}^{2}$ at $\gamma=10$. Also, an extra case was simulated to examine the effect of unequal toughnesses at each $\gamma$ ratio. In cases where $G_{I C}=G_{I I C}$, a similar trend to the linear-softening cohesive zone is observed. However, there is an overall shift towards the local solution for each case. This might be explained by the fact that the developed cohesive zone length will be shorter for a Dugdale type cohesive zone compared to a linear softening cohesive zone for the same toughness and strength properties, again suggesting that the cohesive zone length is the critical parameter. In the cases where $G_{I C}>G_{I I C}$, the predicted partitions also lie between the local and the global solution.

\subsection{2. $A D C B$}

The results of the ADCB test case, modelled with the coupled linear softening cohesive zone, are presented in fig. 7. For the ADCB case, global partitioning predicts that the loading is always pure mode I, regardless of the beam height ratio, and local partitioning predicts a considerable variation in the mode partition as a function of the beam height ratio. The cohesive zone solution again predicts that partitioning lies in between the local and the global solution, and shows a similar trend going from local to global for increasing toughness and decreasing strength. The solutions are again shifted towards the global solution for $\gamma>1$. As the applied moments are equal in this case, the only difference is the geometrical size, which suggests that the smallest characteristic dimension does play an important role in determining the mode mixity. The smallest characteristic dimension is $0.6 \mathrm{~mm}$ in the $\gamma=5$ case compared to $3 \mathrm{~mm}$ in the $\gamma=0.2$ case.

\subsection{Discussion}

Based on the numerical findings presented in section 4.3 , it is evident that the cohesive zone length $\left(l_{c z}\right)$ plays an important role in determining the mode mixity. In the elastic cases where there is no damage (i.e. a zero cohesive zone length), the local partitioning solution provides an accurate estimate of the mode partition and therefore forms a natural lower bound. As the cohesive toughness increases and/or the cohesive strength decreases, the mode partition deviates away from the local solution and moves towards the global solution. For very high damage lengths, the global solution provides an accurate prediction of the mode partition, and hence seems to provide an upper bound. Another important factor in determining the mode mixity, as is evident in the noticeable shift towards the global solution for $\gamma>1$ in the AFRMM and ADCB test cases, is the value of the smallest substrate thickness, termed $a_{c}\left(a_{c}=\min \left(h_{1}, h_{2}\right)\right)$. An overall plot of the normalised mode mixity against the normalised cohesive zone length for all of the test cases is shown in fig. 8. The mode mixity is normalised with respect to the upper and lower bounds of the global and local solutions as:

$$
f=\frac{\left(\frac{G_{I I}}{G}\right)-\left(\frac{G_{I I}}{G}\right)_{W}}{\left(\frac{G_{I I}}{G}\right)_{H S}-\left(\frac{G_{I I}}{G}\right)_{W}}
$$

where $\left(\frac{G_{I I}}{G}\right)_{H S}$ and $\left(\frac{G_{I I}}{G}\right)_{W}$ are the partitions predicted by the local (eq. 5 and global (eq. 3 analytical approaches respectively. The measured cohesive zone length is normalised with respect to the smallest 
characteristic dimension to obtain a normalised damage length parameter $\left(l_{n d}=l_{c z} / a_{c}\right)$. Based on the results in fig. 8, there is strong evidence to suggest a unique dependency exists which describes the normalised mode mixity as a function of the normalised cohesive zone length, which is independent of applied moment ratio $k$, beam height ratio $\gamma$ and cohesive zone type. A best fit exponential curve is fitted to the data which results in the relation:

$$
f= \begin{cases}1, & \text { if } l_{n d} \leq 0.3 \\ 0.9682 e^{-0.24 l_{n d}}+0.0983 e^{-0.02 l_{n d},}, & \text { otherwise }\end{cases}
$$

Based on this fit, the local partitioning is accurate for cohesive zone lengths up to $30 \%$ of the smallest characteristic dimension. This length is considerably larger than the size of the $K$ dominant region, which is reported in Becker et al. 13 and Charalambides et al. 26] to be of the order of $1 \%$ of the beam thickness in beam-like geometries subjected to bending. This result is in agreement with Parmigiani and Thouless [27]; though most cohesive zone lengths based on the current properties fall outside of this $30 \%$ bracket, which suggest that the local partitioning will in general not be accurate for partitioning fractures in composites and adhesive joints. Global partitioning does not in general provide accurate partitioning either, and in most general cases the actual partition lies between the local and global solutions. In general, the mixed mode partition is dependent on multiple cohesive zone and substrate properties, all of which are linked through a cohesive length scale. Taking the unique dependency observed in fig. 8, it is proposed that if a reasonable estimate of the numerical cohesive zone length can be obtained analytically from known substrate and cohesive properties, then an accurate estimate of the mode mixity can be obtained directly from the empirical curve given by eq. 15, and therefore avoiding the need to carry out numerical mixed mode cohesive zone simulations. This idea forms the basis of the semi-analytical cohesive analysis, which is outlined in the following section.

\section{Semi-Analytical Cohesive Analysis (SACA)}

\subsection{Introduction}

In the previous section, the numerical mode mixity is studied as a function of cohesive properties in common asymmetric fracture test geometries and it is found that a unique curve defines the numerical mode mixity as a function of the normalised cohesive zone length (i.e. eq. 15). In this section, a semianalytical approach (SACA) is proposed which makes use of this observed unique curve and hence provides an approximate approach which does not require each individual fracture test to be modelled numerically with cohesive zones in order to determine the correct mode mixity. This greatly reduces the time required to analyse mixed mode fractures and eliminates the need to carry out a numerical analysis. The underlying idea is that if the cohesive zone length can be estimated analytically from the assumed substrate and cohesive properties, then the mode mixity can be obtained directly from the empirically measured unique curve (eq. 15). The analytical estimation of cohesive zone lengths is presented in the following section.

\subsection{Analytical estimation of cohesive zone length}

Yang and Cox [25] presented analytical expressions for pure mode I $\left(l_{I_{-} c z}\right)$ and pure mode II $\left(l_{I I_{-} c z}\right)$ cohesive zone lengths in symmetric slender laminates as:

$$
\begin{gathered}
l_{I_{-} c z}=M\left(E_{I}^{\prime} \frac{G_{I C}}{\left(t_{N C}\right)^{2}}\right)^{\frac{1}{4}} h^{\frac{3}{4}} \\
l_{I I_{-} c z}=M \sqrt{\left(E_{I I}^{\prime} \frac{G_{I I C}}{\left(t_{S C}\right)^{2}} h\right)}
\end{gathered}
$$


where $M$ is a scaling factor, which is included here to account for cohesive zone shape, as in [28; $h$ is half the specimen height and $E_{I}^{\prime}$ and $E_{I I}^{\prime}$ are elastic constants, which are given in [29] for slender orthotropic specimens as:

$$
\begin{aligned}
\frac{1}{E_{I}^{\prime}} & =\sqrt{\frac{1}{2 E_{11} E_{22}}\left(\left(\frac{E_{11}}{E_{22}}\right)^{\frac{1}{2}}+\frac{2\left(\frac{-v_{21}}{E_{22}}\right)+\frac{1}{G_{12}}}{2\left(\frac{1}{E_{11}}\right)}\right)} \\
\frac{1}{E_{I I}^{\prime}} & =\frac{\left(1-v_{12} v_{21}\right)}{E_{11}}
\end{aligned}
$$

where $E_{11}$ and $E_{22}$ are the Youngs moduli in the $x$ and $y$ directions respectively for the loading configurations presented in fig. 4. $G_{12}$ is the in plane shear modulus, and $v_{12}, v_{21}$ are the in-plane Poisson's ratios. The accuracy of eq. $16 \mathrm{a}$ and $16 \mathrm{~b}$ are studied in detail in 28 for mid-plane delaminations under pure mode I and II loadings: it is found that they provide reasonable accuracy for a coupled bilinear cohesive zone when $M=0.5$. To the best of the authors' knowledge, the accuracy of the expressions have not yet been tested for asymmetric geometries, or in their ability to predict cohesive lengths under general mixed mode conditions. Firstly, to deal with the potential asymmetry of geometry and loading, it is proposed that the $h$ term in eq. 16 be defined as:

$$
h=\frac{h_{1}+|k| h_{2}}{1+\frac{\max \left[h_{1}, h_{2}\right]}{\min \left[h_{1}, h_{2}\right]}|k|}
$$

where $k$ is the applied moment ratio as defined in fig. 1 for $-1 \leq k \leq 1$. This proposed definition of $h$ is based on the observation that the estimated cohesive zone length is dependent only on the thickness of the loaded arm in the AFRMM test case $(k=0)$, and is dependent on the minimum arm thickness in the ADCB test case $(k=-1)$. The mode I and mode II cohesive zone lengths under mixed mode conditions $\left(l_{I_{-} c z}^{\prime}, l_{I I_{-} c z}^{\prime}\right)$ can be estimated by replacing $G_{I C}$ with $G_{I}$ in eq. $16 \mathrm{a}$ and $G_{I I C}$ with $G_{I I}$ in eq. $16 \mathrm{~b}$. This results in a mode I and a mode II cohesive zone length defined by:

$$
\begin{aligned}
& l_{I_{-} c z}^{\prime}=M\left(E_{I}^{\prime} \frac{G_{I}}{\left(t_{N C}\right)^{2}}\right)^{\frac{1}{4}} h^{\frac{3}{4}} \\
& l_{I_{-} c z}^{\prime}=M \sqrt{\left(E_{I I}^{\prime} \frac{G_{I I}}{\left(t_{S C}\right)^{2}} h\right)}
\end{aligned}
$$

The largest of these predicted values is then taken to be the cohesive zone length, as depicted in fig. 3

$$
l_{c z}=\max \left[l_{I_{-} c z}^{\prime}, l_{I I_{-} c z}^{\prime}\right]
$$

In order to obtain values of $M$ for the linear softening and Dugdale cohesive zones, the measured cohesive zone lengths from the numerical test cases carried out in section 4 are plotted against the predicted cohesive zone lengths obtained from eqs. 17| 20 for each case, $M$ is varied until an optimum least squares fit between the measured vs. predicted data and the theoretical linear prediction $(x=y)$ is obtained. The measured vs. predicted cohesive zone lengths for the linear softening cohesive zone are plotted in fig. 9(a) $M=0.65$ was found to give the optimum prediction. The measured vs. predicted cohesive zone lengths for the Dugdale cohesive zone are plotted in fig. $9(\mathrm{~b})$ in this case, $M=0.33$ was found to give the best prediction. Interestingly, Planas and Elices [30 previously reported scaling factors of 0.731 for the linear softening cohesive zone and $\pi / 8$ for the Dugdale cohesive zone, based on an analytical study of cohesive zone lengths in infinite bodies. These values are in line with the current predictions for the scaling factors in slender specimens. Overall, the proposed equations are shown to give reasonable estimates for both the linear softening (using $M=0.65$ ) and Dugdale (using $M=0.33$ ) cohesive zones, over a range of cohesive properties, beam height ratios and moment ratios. 


\subsection{Estimating mode mixity}

Once a reasonable estimate of the cohesive zone length is obtained analytically, the normalised mode mixity, $f$, can be estimated directly from the unique dependency curve (eq. 15). The value of $f$ can then be substituted directly into eq. 14 to obtain an estimate of the mode mixity $\left(\frac{G_{I I}}{G}\right)$. In order to obtain the mixed mode cohesive zone lengths from eq. 19, $G_{I}$ and $G_{I I}$ must first be estimated. In the estimation of the cohesive zone lengths shown in figs. 9(a) and 9(b), the values of $G_{I}$ and $G_{I I}$ were obtained directly from the numerical results; however, as the mode mixity will not generally be known prior to the analysis of experimental data, the values of $G_{I}$ and $G_{I I}$ must be found iteratively. To do this, an initial guess of $f$ is made, which gives a mode mixity value from eq. 14. This value of mode mixity is used to partition $G_{c}$ to obtain $G_{I}$ and $G_{I I}$. The value of $G_{c}$ is either known directly from experiments, or can be estimated from a failure locus, if known. The estimated cohesive zone length is then used to obtain an improved estimate of $f$ from eq. 15, and the process is iterated until a converged value of cohesive zone length, and hence mode mixity, is obtained (a tolerance value $(t o l)$ of $1 e-5$ is used in the current work). This process is outlined in fig. 10

\subsection{Numerical Verification}

The proposed SACA approach outlined in fig. 10 is tested here in its ability to predict the numerical damage length and mode partition for general numerical cases using the coupled linear softening cohesive zone. The AFRMM (fig. 4(a) test case is used as before but with a number of changes to the substrate and cohesive properties. The substrate Young's modulus is set to $75 \mathrm{GPa}$ and the tests are carried out at $\gamma=$ $0.1,0.2,0.5,2,5,10$. For each $\gamma$ ratio, five different cohesive toughnesses are tested corresponding to 250 , $500,1000,2000$ and $3000 \mathrm{~J} / \mathrm{m}^{2}$. A linear failure locus is again assumed, with $G_{I C}=G_{I I C}$. In each case the cohesive strength is set to $t_{N C}=t_{S C}=45 \mathrm{MPa}$. The test cases are simulated in Abaqus as before and the mode partition is calculated using the mode decomposed $J$ integral (eq. 13), and the cohesive lengths are recorded. As an aside, it is possible to check the validity of the unique empirical curve proposed in eq. 15 using the new numerical data; to do this, the normalised mode mixity is plotted against the normalised damage length for each of the new simulations in fig. 11. It can be seen that the proposed empirical curve accurately describes the normalised mode mixity as a function of the normalised cohesive zone length for the new numerical data, and hence further suggests that the measured empirical curve is unique.

Once the cohesive zone lengths and mode partitions are recorded from the numerical solution, the required parameters are independently input into the SACA analysis in order to estimate the cohesive zone lengths and mode partitions. The required parameters are: geometry and loading conditions $\left(h_{1}, h_{2}, k\right)$; substrate elastic properties $\left(E_{11}, E_{22}, G_{12}, v_{12}, v_{23}\right)$; cohesive strengths $\left(t_{N C}, t_{S C}\right)$ and cohesive zone shape $(M)$. In the SACA partitioning of this numerical case, $G_{c}$ is found from the assumed failure locus at a given mode mixity. The SACA predicted damage lengths are plotted against the numerically obtained damage lengths in fig. 12(a), and the SACA predicted mode partitions are plotted against the numerically obtained mode partitions in fig. 12(b) There is some small scatter in the prediction of the damage length in fig. 12(a) though the general trend is accurately predicted. This suggests there may be scope for improvement of the analytical cohesive zone length estimation. However, the predicted mode partitions in fig. 12(b) are in very good agreement with the numerically obtained mode partition. This suggests that the mode partition is not overly sensitive to small scatter in the cohesive length estimation, and that the semi-analytical model is sufficient to gain a good estimate of the mode partition in the general case. This SACA approach therefore eliminates the need to numerically model each case individually and makes it feasible to estimate the mode mix efficiently based on substrate and cohesive properties. In the following section, this semi-analytical cohesive analysis is applied to a number of experimental case studies taken from literature.

\section{Experimental Case Studies}

\subsection{Hashemi et al.}

Two case studies from the literature are analysed in this section. The first is on the AFRMM delamination tests carried out by Hashemi et al. [1] on uni-directional carbon fibre epoxy composite. In this original work, 
it was found that global partitioning produced the most physical failure locus when applied to partition the asymmetric test data. It was therefore suggested that global partitioning should be used to partition fractures in such material systems. Using the data presented in this paper, it was possible to obtain the beam height ratios and dimensions, and re-partition the data according to the SACA approach. Typical uni-directional carbon fibre epoxy elastic properties were assumed as they were not presented in the paper. The values used were $E_{11}=120 \mathrm{GPa}, E_{22}=10 \mathrm{GPa}, G_{12}=4.6 \mathrm{GPa}, v_{12}=0.3, v_{23}=0.38$. The values of $G_{c}$ at each height ratio are taken directly from the experimental measurements presented in the paper. No data was available for the cohesive strength, so a typical value of $45 \mathrm{MPa}$ was assumed in normal and shear. Finally, a value of the shape parameter $M$ must be chosen; Svensson et al. 31] recently measured mode I and mode II cohesive zone laws in carbon fibre reinforced composite and found that a trapezoidal law accurately described the stress as a function of opening displacement. Therefore, both the linear softening $(M=0.65)$ and Dugdale $(M=0.33)$ cases are used here in the SACA analysis as they give an upper and lower bound for the trapezoidal shape. The predicted partitions according to the SACA approach are presented in fig. 13(a) for both the linear softening (L) and Dugdale (D) cohesive zones.

In all cases, the SACA partitioning predicts that the partition lies between the local and the global partitioning. For $\gamma<1$, the partitioning is closer to the local analysis, and as $\gamma$ becomes large and mode II becomes dominant, the partitioning approaches the global solution. As expected, the linear softening cohesive zone partitioning lies closer to the global partitioning compared to the Dugdale partitioning; this is due to the larger cohesive zone length prediction in the linear softening case. The predicted failure loci based on the linear softening and Dugdale partitioning are presented in fig. 13(b). These are also compared to the local and global partitioning of the data, which were presented in the original paper. In order to obtain a quantitative assessment of the accuracy of each of the methods, a linear failure locus is assumed to be correct and the root mean square errors ( $r m s e)$ are calculated for each partitioning method, where rmse is defined as:

$$
\text { rmse }=\sqrt{\frac{\sum_{1}^{N}\left(\frac{G_{\text {curve }}-G_{\text {data }}}{G_{\text {data }}}\right)^{2}}{N}}
$$

where $N$ is the number of data points. The rmse values are presented in fig. 13(b) it is evident that the SACA (L) partitioning produces the best fit to the assumed linear failure locus. As previously reported, it is also evident from the rmse values that the local partitioning does not produce overall accurate partitioning for this material system. Interestingly, fig. 13(a) indicates that the local partitioning is reasonably accurate for $\gamma<1$ based on the SACA analysis, but not for $\gamma>1$. While SACA (L) partitioning produces the best fit, SACA (D) partitioning is also compatible with the experimental results, and also produces a lower rmse than global partitioning. The ability of the SACA approach to predict a physical failure locus is promising and provides direct experimental evidence which demonstrates that the cohesive length scale can be used to predict partitioning based on the numerically observed unique dependency between local and global partitioning solutions.

\subsection{Ducept et al.}

The second case study is based on experimental data of Ducept et al. [15]. In this paper, ADCB and asymmetric mixed mode bend (AMMB) test cases were carried out on glass fibre reinforced epoxy composite for a number of different height ratios and moment ratios respectively. AMMB cases are those in which unequal moments are applied to the substrate arms. The asymmetric tests were then partitioned according to the global and local approaches, and the resulting failure loci were compared to the true failure locus, which was measured using symmetric specimens. It was found that local partitioning accurately predicted the symmetrically measured failure locus, while the global partitioning did not perform well in predicting the true failure locus. It was therefore deemed that local partitioning provided the most accurate partitioning for this material system. The ADCB and AMMB data of Ducept et al. 15] are re-partitioned here using the SACA approach. The glass fibre reinforced epoxy composite elastic properties are given as $E_{11}=25.7 \mathrm{GPa}$, $E_{22}=6.5 \mathrm{GPa}, G_{12}=2.5 \mathrm{GPa}, v_{12}=0.32$ and $v_{23}=0.38$. The values of $G_{c}$ for each test case are reported in the paper. There was no data given on the cohesive strength, so a typical value of $45 M P a$ was again 
assumed in normal and shear. As before, both the linear softening $(M=0.65)$ and Dugdale $(M=0.33)$ cohesive zone shapes are used, as they give upper and lower bound results for various trapezoidal cohesive zone shapes. The predicted SACA partitioning, for both linear softening and Dugdale cohesive zones, are presented in fig. 14(a) for the ADCB test cases, and in fig. 14(b) for the AMMB test.

It is evident that the SACA partitioning for both the ADCB and AFRMM, for linear softening and Dugdale cohesive zones, is very close to the local partitioning for this material system. The predicted failure loci based on these partitioning results are presented in fig. 15. Also presented in fig. 15 are the predicted failure loci according to the local and global partitioning, and the symmetrically measured failure locus. As outlined in Ducept et al. [15, the local partitioning produces a failure locus close to the symmetrically measured locus, while it is evident that the global partitioning is not compatible with the results. The SACA partitioning, using both the linear softening and Dugdale cohesive zones, are also compatible with the experimental results. The local partitioning and SACA(D) perform best when comparing the root mean square error (eq. 21). However, there are only relatively small differences between the resulting failure loci between $\mathrm{SACA}(\mathrm{D})$ and $\mathrm{SACA}(\mathrm{L})$ partitioning in this case. It is believed that due to the relatively low substrate modulus in this case $(25.7 \mathrm{GPa})$, the resulting cohesive length scales are small and hence the predicted SACA partitioning is in good agreement with local partitioning. This prediction, which again is in agreement with the experimental results, further demonstrates that the cohesive zone length scale approach can be used to accurately predict mode partitioning.

\section{Conclusions}

In the first part of this paper, the numerical mixed mode partition in common asymmetric fracture test geometries is studied as a function of varying cohesive properties. The numerical mixed mode partition is calculated using the mode decomposed $J$ integral approach [19]. The main conclusions drawn from this study are:

- When the fracture process zone is small, the resulting value of the numerical mode partition is close to the local partitioning solution. Also in a number of cases where no damage is allowed to develop, the local solution is also recovered, suggesting that this forms a lower bound.

- As the cohesive zone size grows, the values of the numerical mixed mode partition deviate away from the local solution, and in all cases moves towards the global solution which seems to form the upper bound.

- It is found that in most cases the numerical mixed mode partition lies between the local and global solutions, suggesting limited applicability of both the local and global approaches in their current form.

- When the normalised cohesive length for all cases are plotted against the normalised mode mixity, a unique dependency is observed which is independent of test geometry, cohesive properties, and cohesive zone type.

Based on this observed unique dependency, the semi-analytical cohesive analysis (SACA) is proposed for calculating mixed mode partitions. This approach eliminates the need to carry out a numerical analysis and therefore greatly reduces the time and effort required to calculate mixed mode partitions. The method utilises the analytical estimations of cohesive zone length [25] to determine the mode mixity directly from the numerically obtained unique dependency curve (i.e. eq. 15). The method is found to work well in predicting mixed mode partitions when compared to numerically obtained values in a verification test case using the coupled linear softening cohesive zone. The proposed unique dependency curve is also found to hold true for this verification test case.

In the final part of this paper, the proposed SACA method is used to re-partition AFRMM and $\mathrm{ADCB} / \mathrm{AMMB}$ test data from previously contrasting reports in the literature [1, 15. Both linear softening and Dugdale cohesive zone shape factors are used as they give an upper and lower bound to the trapezoidal law. It is found that the SACA partitioning (Linear softening and Dugdale) could be used to 
explain the results from both cases, based on the estimated cohesive length scale parameter. Two main conclusions can be drawn from this:

- The SACA approach captures the experimentally observed shift from the local towards the global partitioning as the level of damage increases.

- The SACA approach proves an efficient and accurate method for predicting mixed mode partitions.

\section{Acknowledgements}

The financial support of Henkel Ireland Ltd. and the Irish Research Council is gratefully acknowledged. The SFI/HEA Irish Centre for High-End Computing (ICHEC) are also gratefully acknowledged for the provision of computational facilities and support. The authors would also like to thank Prof. M.D. Thouless for providing the UEL subroutine for the Uncoupled Dugdale CZM.

\section{References}

[1] S. Hashemi, A. J. Kinloch, J. G. Williams, Mixed-mode fracture in fiber-polymer composite laminates, Composite Materials: Fatigue and Fracture ASTM STP 1110, T.K O'Brien, Ed., American Society for Testing and Materials, Philadelphia 3 (1991) 143-68.

[2] M. Benzeggagh, M. Kenane, Measurement of mixed-mode delamination fracture toughness of unidirectional glass/epoxy composites with mixed-mode bending apparatus, Composites Science and Technology 56 (1996) $439-49$.

[3] D. A. Dillard, H. K. Singh, D. J. Pohlit, J. M. Starbuck, Observations of Decreased Fracture Toughness for Mixed Mode Fracture Testing of Adhesively Bonded Joints, Journal of Adhesion Science and Technology 23 (2009) 1515-30.

[4] M. Conroy, B. F. Sørensen, A. Ivankovic, Combined Numerical and Experimental Investigation of Mode-Mixity in Beam Like Geometries, in: Proceedings of the 37th annual meeting of the Adhesion Society, February 23-26, San Diego, CA, 2014.

[5] ISO 15024:2001, Fibre-reinforced plastic composites Determination of mode I interlaminar fracture toughness, $G_{I C}$, for unidirectionally reinforced materials, 2001.

[6] ISO 15114:2014, Fibre-reinforced plastic composites Determination of the mode II fracture resistance for unidirectionally reinforced materials using the calibrated end-loaded split (C-ELS) test and an effective crack length approach, 2014.

[7] ASTM Standard D 6671-01, Standard Test Method for Mixed Mode I Mode II Interlaminar Fracture Toughness of Unidirectional Fiber Reinforced polymer Matrix Composites, 2001.

[8] L. Banks-Sills, M. Arcan, Y. Bortman, A mixed mode fracture specimen for mode II dominant deformation, Engineering Fracture Mechanics 20 (1984) 145-57.

[9] B. F. Sørensen, K. Jørgensen, T. K. Jacobsen, R. C. Østergaard, DCB-specimen loaded with uneven bending moments, International Journal of Fracture 141 (2006) 163-76.

[10] J. G. Williams, On the calculation of energy release rates for cracked laminates, International Journal of Fracture 36 (1988) 101-19.

[11] W.-L. Yin, J. T. S. Wang, The Energy-Release Rate in the Growth of a One-Dimensional Delamination, Journal of Applied Mechanics 51 (1984) 939-41.

[12] Z. Suo, J. Hutchinson, Interface crack between two elastic layers, International Journal of Fracture 43 (1990) 1-18.

[13] T. Becker, J. McNaney, R. Cannon, R. Ritchie, Limitations on the use of the mixed-mode delaminating beam test specimen: Effects of the size of the region of K-dominance, Mechanics of Materials 25 (1997) $291-308$.

[14] B. D. Davidson, P. L. Fariello, R. C. Hudson, V. Sundararaman, Accuracy Assessment of the Singular-Field Based ModeMix Decomposition Procedure for the Prediction of Delamination, Composite materials: Testing and Design, ASTM STP 1242, S.J. Hooper, Ed., American Society for Testing and Materials 13 (1997) 109-28.

[15] F. Ducept, D. Gamby, P. Davies, A mixed-mode failure criterion derived from tests on symmetric and asymmetric specimens, Composites Science and Technology 59 (1999) 609-19.

[16] D. Dugdale, Yielding of steel sheets containing slits, Journal of the Mechanics and Physics of Solids 8 (1960) $100-4$.

[17] G. I. Barenblatt, The Mathematical Theory of Equilibrium Cracks in Brittle Fracture, Advances in Applied Mechanics Volume 7 (1962) 55-129.

[18] J. R. Rice, A Path Independent Integral and the Approximate Analysis of Strain Concentration by Notches and Cracks, Journal of Applied Mechanics 35 (1968) 379-86.

[19] H. Ishikawa, H. Kitagawa, H. Okamura, J integral of a mixed mode crack and its application, in: K. J. M. SMITH (Ed.), Mechanical Behaviour of Materials, Pergamon, 1980, pp. 447-55. URL: http://www.sciencedirect.com/science/ article/pii/B9781483284149501371 doi http://dx.doi.org/10.1016/B978-1-4832-8414-9.50137-1

[20] A. Turon, C. Dávila, P. Camanho, J. Costa, An engineering solution for mesh size effects in the simulation of delamination using cohesive zone models, Engineering Fracture Mechanics 74 (2007) 1665 -82.

[21] P. Camanho, C. Dávila, M. De Moura, Numerical simulation of mixed-mode progressive delamination in composite materials, Journal of Composite Materials 37 (2003) 1415-38. Cited By 282. 
[22] Q. D. Yang, M. D. Thouless, S. M. Ward, Numerical simulations of adhesively-bonded beams failing with extensive plastic deformation, Journal of the Mechanics and Physics of Solids 47 (1999) 1337-53.

[23] A. Turon, P. P. Camanho, J. Costa, C. G. Dávila, A damage model for the simulation of delamination in advanced composites under variable-mode loading, Mechanics of Materials 38 (2006) 1072-89.

[24] C. Sarrado, A. Turon, J. Renart, I. Urresti, Assessment of energy dissipation during mixed-mode delamination growth using cohesive zone models, Composites Part A: Applied Science and Manufacturing 43 (2012) 2128-36.

[25] Q. Yang, B. Cox, Cohesive models for damage evolution in laminated composites, International Journal of Fracture 133 (2005) 107-37.

[26] M. Charalambides, A. J. Kinloch, Y. Wang, J. G. Williams, On the analysis of mixed-mode failure, International Journal of Fracture 54 (1992) 269-91.

[27] J. Parmigiani, M. D. Thouless, The effects of cohesive strength and toughness on mixed-mode delamination of beam-like geometries, Engineering Fracture Mechanics 74 (2007) 2675-99.

[28] P. W. Harper, S. R. Hallett, Cohesive zone length in numerical simulations of composite delamination, Engineering Fracture Mechanics 75 (2008) 4774-92.

[29] Q. D. Yang, B. N. Cox, R. K. Nalla, R. O. Ritchie, Fracture length scales in human cortical bone: The necessity of nonlinear fracture models, Biomaterials 27 (2006) 2095-113.

[30] J. Planas, M. Elices, Nonlinear fracture of cohesive materials, International Journal of Fracture 51 (1991) $139-57$.

[31] D. Svensson, K. S. Alfredsson, A. Biel, U. Stigh, Measurement of cohesive laws for interlaminar failure of CFRP, Composites Science and Technology 100 (2014) 53-62.

[32] ABAQUS, Version 6.11 documentation, Dassault Systemes Simulia Corp. Providence, RI, USA. (2011).

[33] J. C. Brewer, P. A. Lagace, Quadratic Stress Criterion for Initiation of Delamination, Journal of Composite Materials 22 (1988) 1141-55.

[34] P. P. Camanho, F. L. Matthews, Delamination Onset Prediction in Mechanically Fastened Joints in Composite Laminates, Journal of Composite Materials 33 (1999) 906-27.

[35] J. R. Reeder, An evaluation of mixed-mode delamination failure criteria, NASA technical memorandum 104210. Langley Research Center, Hampton, VA, 1992.

[36] M. Mathews, S. Swanson, Characterization of the interlaminar fracture toughness of a laminated carbon/epoxy composite, Composites Science and Technology 67 (2007) 1489-98.

\section{Appendices}

\section{A. Cohesive Zone Models}

\section{A.1. Coupled Linear-Softening CZM [32]}

The coupled linear-softening cohesive zone, which is inbuilt into Abaqus, is shown graphically in fig. 5(a) The cohesive zone is based on the formulation proposed by Camanho et al. 21]. Up to the point of damage initiation, the response of the cohesive zone is linear and uncoupled in opening and shear and governed by the pre-damage stiffness matrix:

$$
\left[\begin{array}{c}
t_{N} \\
t_{S}
\end{array}\right]=\left[\begin{array}{cc}
k_{y y} & 0 \\
0 & k_{x x}
\end{array}\right]\left[\begin{array}{c}
\delta_{N} \\
\delta_{S}
\end{array}\right]
$$

where $t_{N}$ and $t_{S}$ are the normal and shear tractions respectively acting across the cohesive zone, $\delta_{N}$ and $\delta_{S}$ are the relative displacements of the cohesive zone in the normal and shear directions respectively, and $k_{y y}$ and $k_{x x}$ are the initial penaltly stiffnesses in the normal and shear directions respectively. The initial penalty stiffnesses are set at a value of $1 \mathrm{e} 15 \mathrm{~Pa} / \mathrm{m}$ for all simulations. The cohesive tractions increase linearly with displacement according to eq. 22 until they satisfy the quadratic initiation criterion, which is given by:

$$
\left(\frac{\left\langle t_{N}\right\rangle}{t_{N C}}\right)^{2}+\left(\frac{t_{S}}{t_{S C}}\right)^{2}=1
$$

where $t_{N}$ and $t_{S}$ are the normal and shear tractions respectively acting across the cohesive zone, and $t_{N C}$ and $t_{S C}$ are the chosen pure mode cohesive strengths in normal and shear respectively. The quadratic stress criterion (eq. 23) has been used previously to accurately predict delamination onset in composite laminates [33. 34. After damage initiation, a linear softening law is effected using an overall damage variable $D$, 
which is incremented from zero to one as a function of the overall effective displacement, $\delta_{\text {eff }}$, where $\delta_{e f f}=\sqrt{\delta_{N}^{2}+\delta_{S}^{2}}$, as:

$$
D=\frac{\delta_{e f f}^{f}\left(\delta_{e f f}^{\max }-\delta_{e f f}^{0}\right)}{\delta_{e f f}^{\max }\left(\delta_{e f f}^{f}-\delta_{e f f}^{0}\right)}
$$

where $\delta_{e f f}^{f}$ is the effective displacement at final failure, $\delta_{e f f}^{0}$ is the effective displacement at damage initiation, and $\delta_{e f f}^{\max }$ is the maximum effective displacement that has occurred up to that point in time. The cohesive tractions in the softening regime, which are coupled through $D$, are then computed from

$$
\begin{aligned}
& t_{N}= \begin{cases}(1-D) \bar{t}_{N}, & \text { if } \bar{t}_{N} \geq 0 \\
\bar{t}_{N}, & \text { otherwise }\end{cases} \\
& t_{S}=(1-D) \bar{t}_{S}
\end{aligned}
$$

where $\bar{t}_{N}$ and $\bar{t}_{S}$ are the tractions predicted by the pre-damage stiffness matrix (eq. 22 at the current displacements. Along with pure mode cohesive strengths and penalty stiffnesses, it is also required to specify the mixed mode failure locus. The following linear criterion is used

$$
\left(\frac{G_{I}}{G_{I C}}\right)+\left(\frac{G_{I I}}{G_{I I C}}\right)=1
$$

where $G_{I}$ and $G_{I I}$ are the energies dissipated in mode I and mode II respectively up to that point in time (as represented by the red and blue shaded regions respectively in fig. 5(a)d, and $G_{I C}$ and $G_{I I C}$ are the prescribed pure mode cohesive toughnesses, as indicated in fig. 6(a) The linear failure criterion has been used extensively to describe mixed mode failure loci [35, 36] in composites.

\section{A.2. Uncoupled Dugdale (constant traction) CZM [22]}

The uncoupled Dugdale CZM, which is developed by Yang et al. 22, is shown in fig. 5(b)] Prior to damage initiation, the response of the element is equivalent to the coupled linear softening cohesive zone (i.e. eq. 22 , and the penalty stiffnesses are also both set to $1 \mathrm{e} 15 \mathrm{~Pa} / \mathrm{m}$ in each case. In this formulation, the stresses remain uncoupled and constant as they follow the prescribed pure mode Dugdale type laws exactly until the failure criterion is satisfied, at which point the stress is dropped suddenly to zero in both modes. The linear failure criterion (eq. 26) is also used in this formulation. 


\section{Figures}

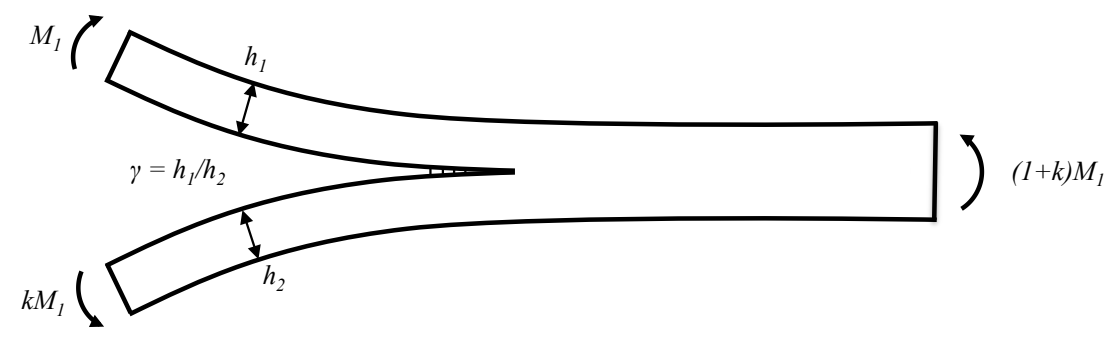

Figure 1: Beam-like geometry subjected to pure bending moments $\left(M_{1}, k M_{1}\right)$.

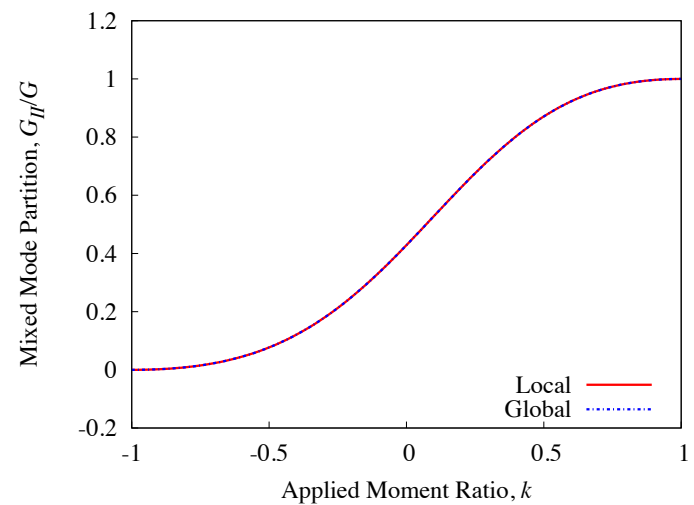

(a) Mixed Mode Bend Specimen ( $\gamma=1, k$ varied). - Both lines may not be visible as they overlap.

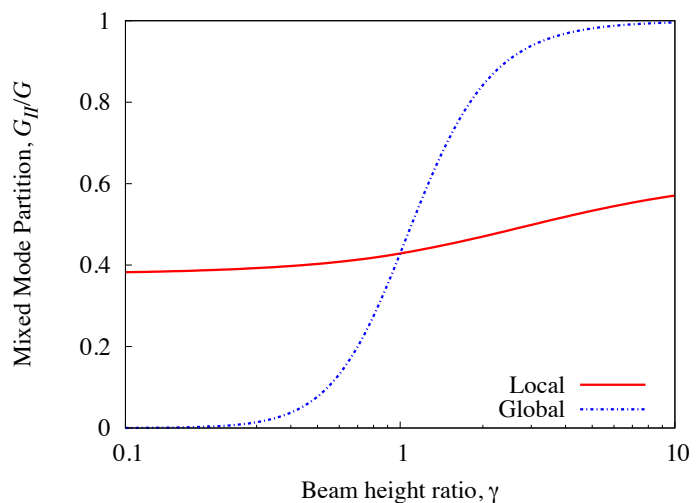

(b) Asymmetric Fixed Ratio Mixed Mode ( $k=0, \gamma$ varied $)$.

Figure 2: Local and Global Partitioning for (a) symmetric specimens, and (b) asymmetric specimens. 


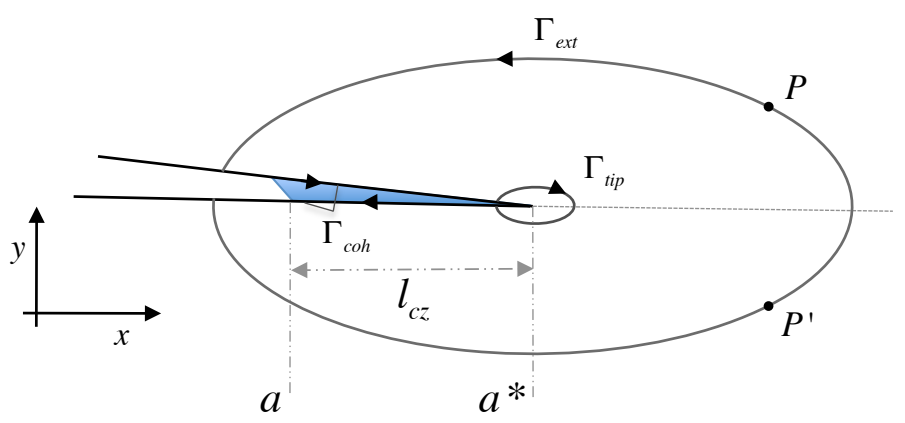

Figure 3: Fracture Process Zone.

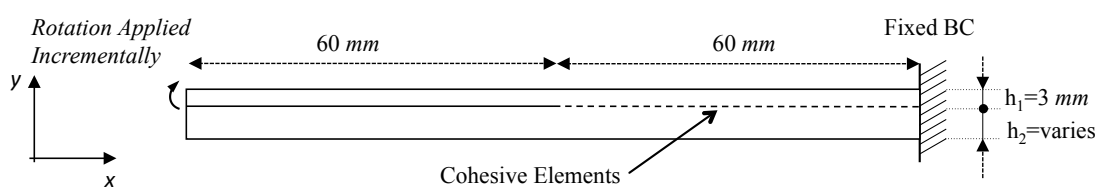

(a) Asymmetric Fixed Ratio Mixed Mode (AFRMM).

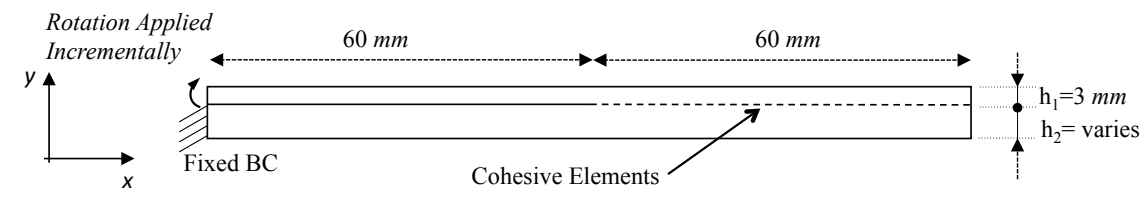

(b) Asymmetric Double Cantilever Beam (ADCB).

Figure 4: Beam Geometries and Loading Conditions.

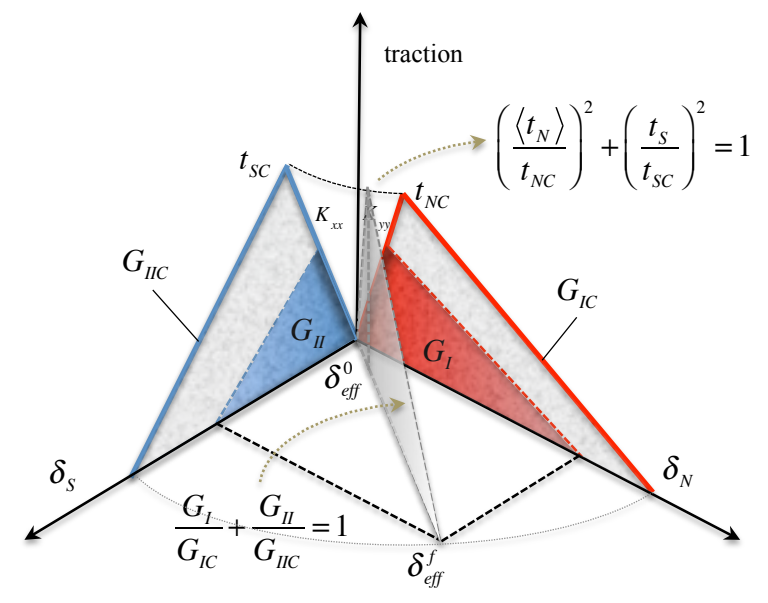

(a) Coupled Linear Softening Cohesive Zone

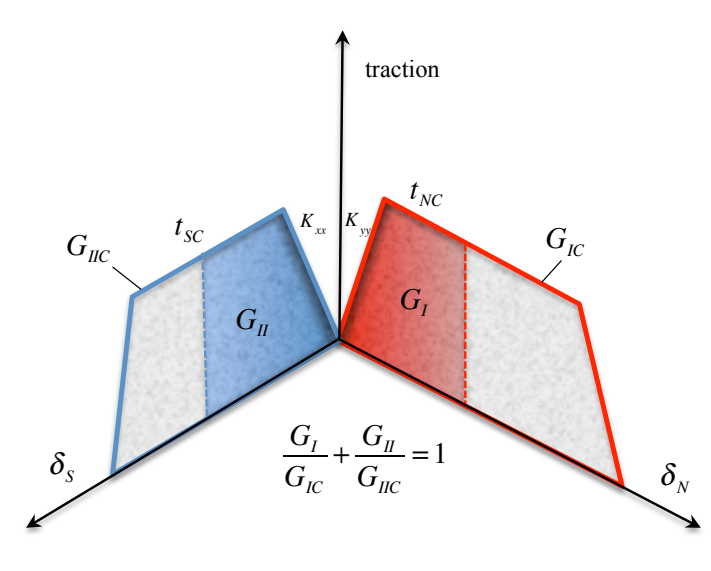

(b) Uncoupled Dugdale Cohesive Zone.

Figure 5: Cohesive Zone Formulations. 


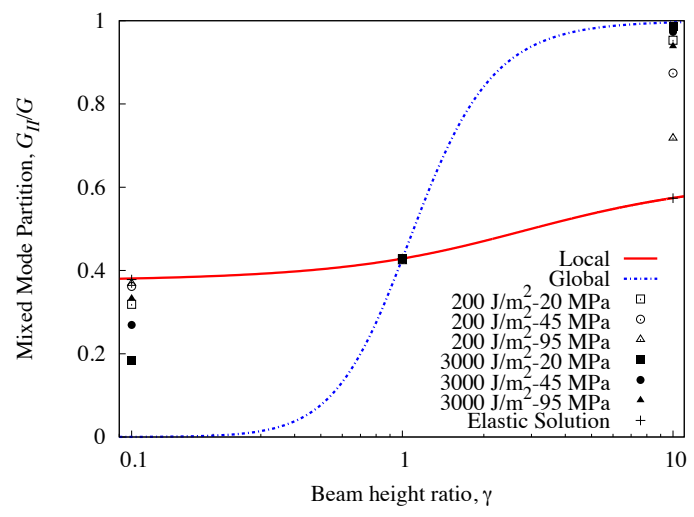

(a) Coupled Linear Softening Cohesive Zone

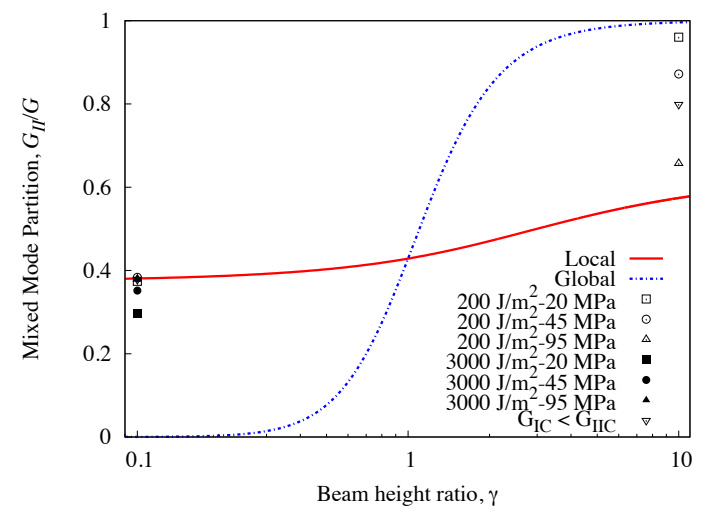

(b) Uncoupled Dugdale Cohesive Zone.

Figure 6: Numerical mode partitions from cohesive zone analysis of the Asymmetric Fixed Ratio Mixed Mode for (a) Coupled Linear-Softening Cohesive Zone and (b) Uncoupled Dugdale Cohesive Zone.

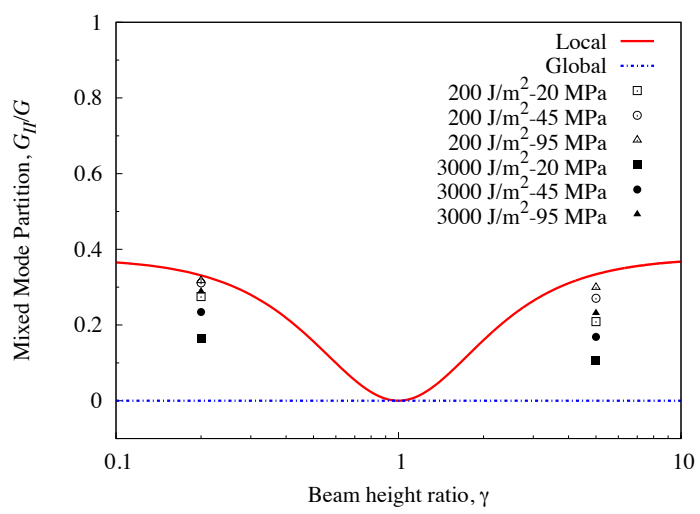

Figure 7: Numerical mode partitions from cohesive zone analysis for the Asymmetric Double Cantilever Beam (Coupled Linear Softening Cohesive Zone). 


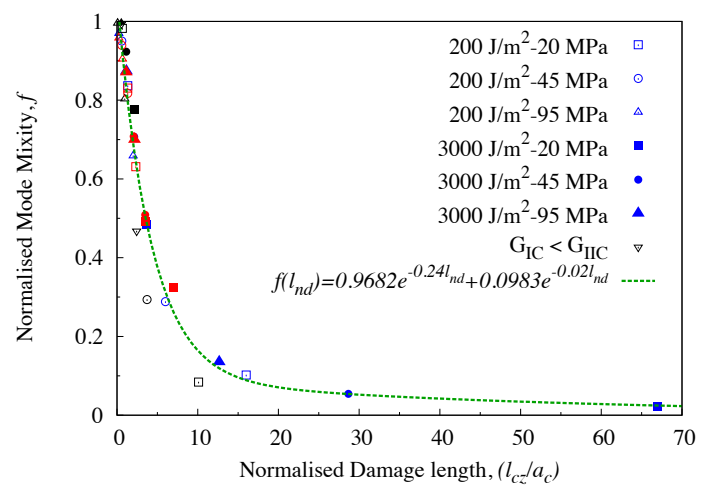

Figure 8: Normalised Mode Mixity vs. Normalised Damage length for AFRMM linear-softening CZM (blue), AFRMM Dugdale CZM (black) and ADCB linear-softening CZM (red).



(a) Coupled Linear Softening Cohesive Zone

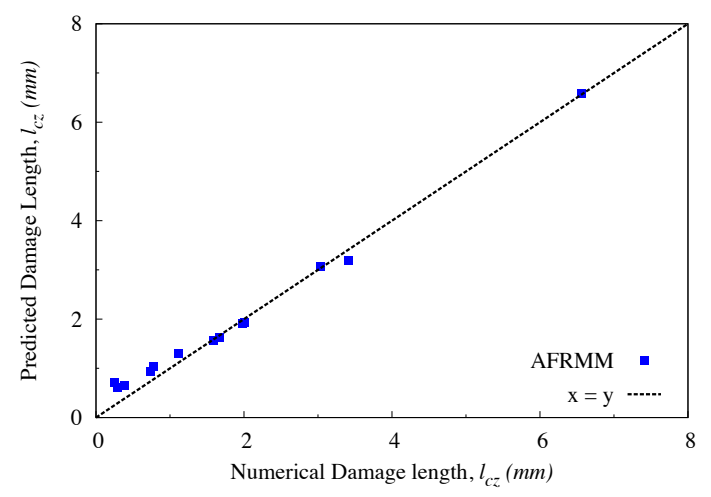

(b) Uncoupled Dugdale Cohesive Zone.

Figure 9: Numerically obtained damage length plotted against analytically predicted damage length for (a) Coupled LinearSoftening Cohesive Zone $(M=0.65)$ and (b) Uncoupled Dugdale Cohesive Zone $(M=0.33)$ 


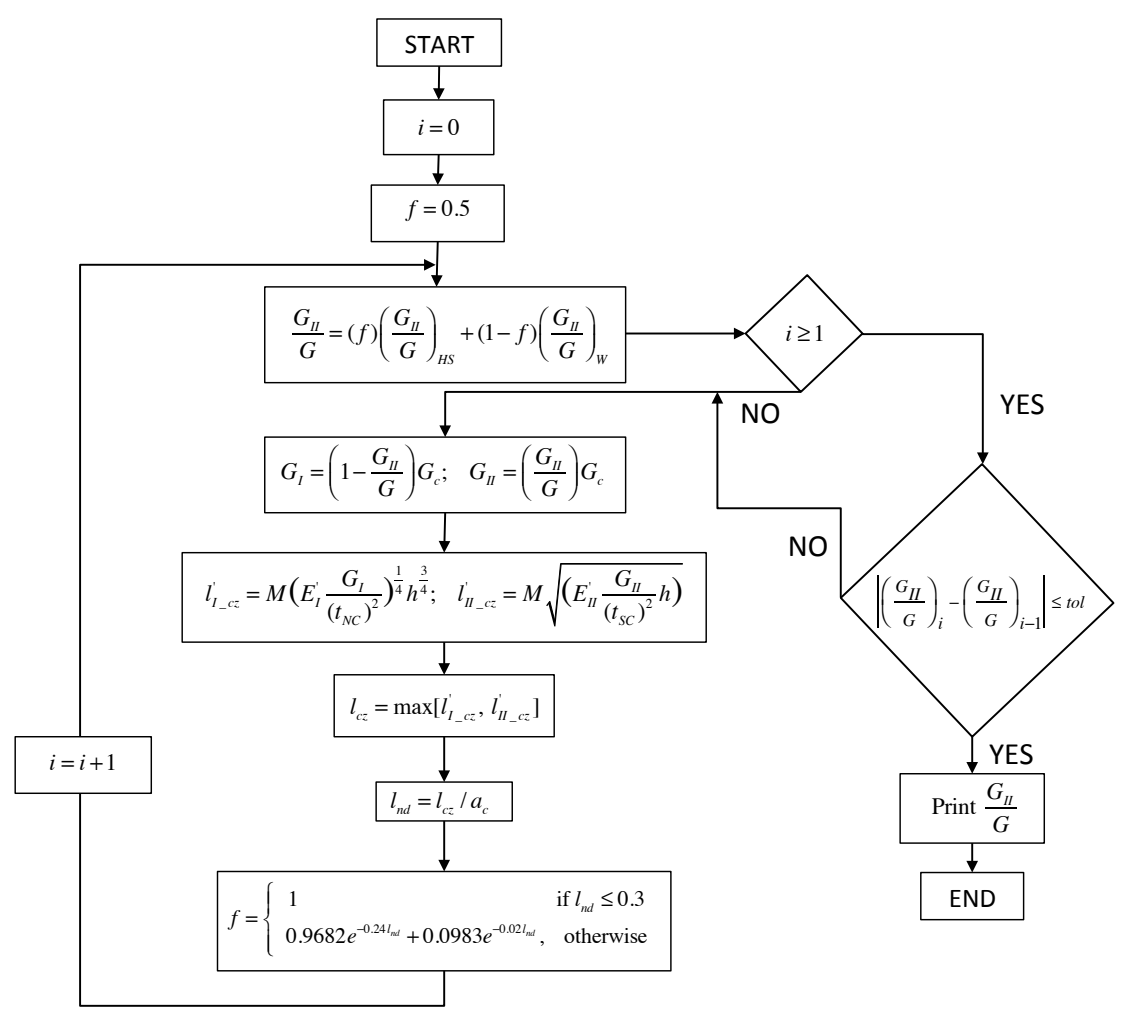

Figure 10: Program flowchart for the proposed semi-analytical cohesive analysis (SACA).



Figure 11: Numerically obtained damage length scale plotted against the normalised mode mixity, $f$, for the AFRMM verification test cases using the linear softening cohesive zone. 


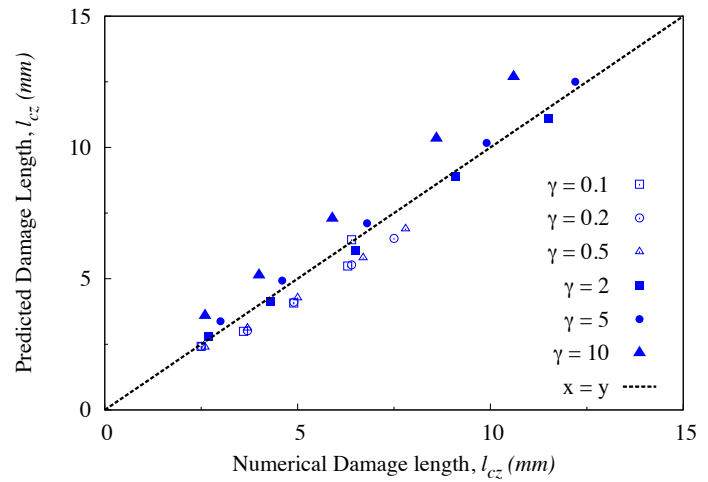

(a) Cohesive Zone Length

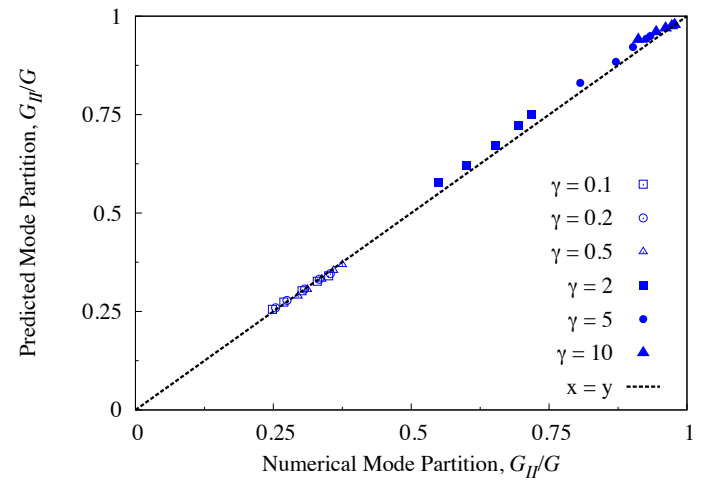

(b) Mixed Mode Partition

Figure 12: Numerically obtained (a) cohesive zone length, and (b) mode mixity, plotted against SACA predicted damage length and mixed mode partition, respectively, for the AFRMM verification test cases using the linear softening cohesive zone.

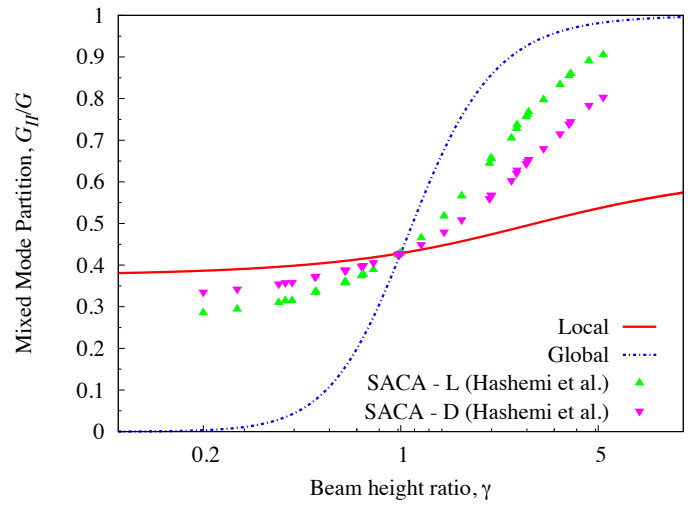

(a) AFRMM Partitioning

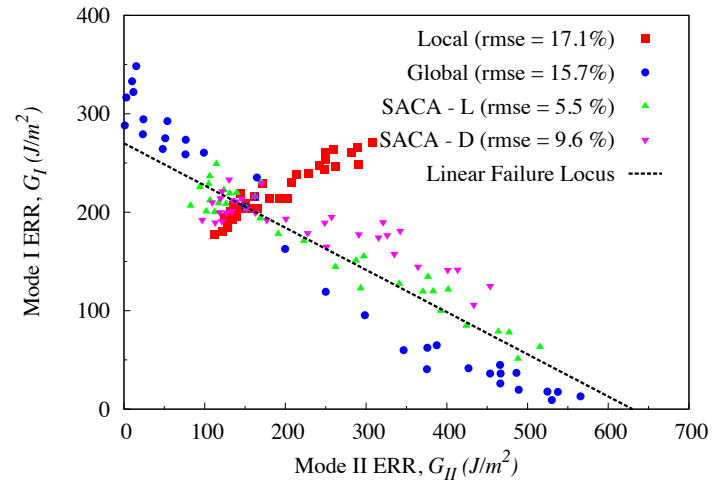

(b) Failure Locus

Figure 13: (a) SACA partitioning (L-linear softening, D-Dugdale) and (b) Predicted failure loci resulting from this partitioning of the AFRMM data obtained in Hashemi et al. [1] 




(a) ADCB Partitioning

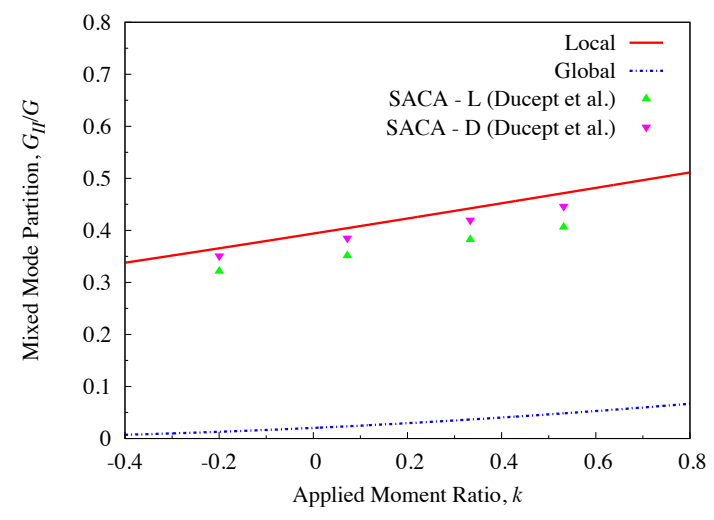

(b) AMMB Partitioning

Figure 14: SACA (L-linear softening, D-Dugdale) partitioning for (a) ADCB test data and (b) AMMB test data obtained from Ducept et al. [15.

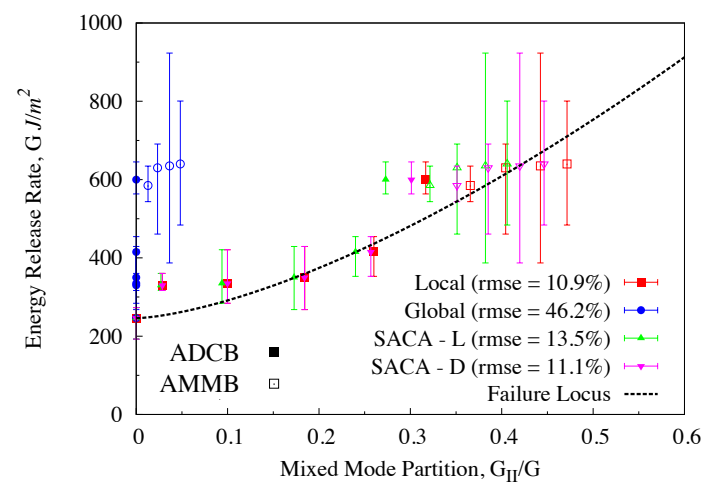

Figure 15: Predicted failure loci resulting from local, global and SACA (L-linear softening, D-Dugdale) partitioning of the $\mathrm{ADCB}$ and $\mathrm{AMMB}$ data obtained in Ducept et al. [15. The predicted failure loci are compared to the symmetrically measured failure locus. 